\title{
Scale-Free Structure in Concept Lattices Associated to Complex Systems
}

\author{
Gonzalo A. Aranda-Corral \\ Department of Information Technology \\ Universidad de Huelva \\ Palos de La Frontera, Spain \\ Email:garanda@us.es
}

\author{
Joaquín Borrego-Díaz \\ Dept. Computer Science and AI \\ Universidad de Sevilla \\ Sevilla, Spain \\ Email:jborrego@us.es
}

\author{
Juan Galán-Páez (*) \\ Dept. Computer Science and AI \\ Universidad de Sevilla \\ Sevilla, Spain \\ Email:juangalan@us.es
}

\begin{abstract}
Qualitative representation and reasoning on Complex Systems (CS) is important for a number of human activities on CS, mainly for the understanding of both, our perception about their structure as well as their dynamics. Formal Concept Analysis can help understanding the conceptual structure behind these qualitative representations by means of the called concept lattices (CL). In this paper the scale free conceptualization hypothesis, (SFCH) is asserted. SFCH claims that a scale-free distribution in node's connectivity appears on the CL associated to complex systems (CLCS) only when two requirements holds: CLCS is useful both to represent qualitative and reliable attributes on the $\mathrm{CS}$, and to provide a basis for (qualitative) successfully reasoning about the CS. Experiments revealed that the topologies of CLCS are similar when the amount of information on the CS is sufficient, while it is different in other concept lattices associated to random formal contexts or to other systems in which some of the above requirements do not hold.

Index Terms-Semantic Networks, Scale Free Conceptualization Hypothesis, Formal Concept Analysis.
\end{abstract}

\section{INTRODUCTION}

An interesting feature in Complex Systems (CS) research is to investigate how humans describe and understand such kind of systems. It is very intriguing how human's rationality is able to select important features and concepts (and the relationships among them) of the CS in order to reason and predict its dynamics (for surviving within) or to describe their features. This paper reports an empirical analysis of semantic networks built to specify the (qualitative) knowledge retrieved from CS. Semantic Networks represent an useful tool to represent specific features of CS as for example language evolution and structure (see e.g. [13]), but their scope may include other CS where the analysis of semantic relationships among concepts involved in CS is necessary. General (formal) representations of Semantic relationships are often complex networks as well, and their analysis gives some insights on the complex nature of CS whose features are represented.

The above approach to qualitative representation and reasoning on CS is ambitious, because the idea of CS covers very different systems, with an astonishing variety of dynamics. In the representation of CS, it is necessary to choose what features the observer thinks essential to understand it, and how these features are related. Human reasoning activities are essential to understand system's behavior, and they strongly depends on our ability to quickly select key features and to reason with limited resources. However, this reasoning can not use every existent relationship among features; only few ones the user thinks as important (a particular bounded rationality strategy, as for example [11]). However, the global structure which comprises all the features, concepts and their relationship, represents a complex structure which can give more information on the CS than user consequences. Examples where interest on the global complex structures requires more attention are stock markets, economy, ontology/folksnomy evaluation, betting markets and, in general, those CS in which humans are involved, and where their decisions are based on partial information on the CS. To model this semantic network, Formal Concept Analysis has been selected.

Formal Concept Analysis (FCA) [10] is a mathematical theory for data analysis whose basic data structure is the formal context which consists on a set of objects and their properties. They represent weak structures easily built from experience that allow the extraction of knowledge. Despite its simple data structure, formal contexts are useful structures for knowledge extraction and reasoning (cf. [10]). When FCA is applied on a considerable amount of observable features of the CS, the Concept Lattice (CL) generated includes every concept involved in CS description; it represents a complex (semantic) network. Concepts and relationships within the CL can describe features of the CS as well as they can aid the observer to work with the information (for describing, classifying, predicting, etc.).

The aim of this paper is twofold. On the one hand, we discuss the main issues related with concept lattices associated to observations on CS. We will see how the analysis of topological features of CLCS, considered as complex networks, may aid understanding CS evolution in some cases. On the other hand we assert that when the observations are objective and relevant in order to study the CS, the associated CL exhibits a scale-free distribution structure. This claim (which we call scale-free conceptualization hypothesis, SFCH) is experimentally analyzed in a number of examples.

The structure of the paper is as follows. The next section is 


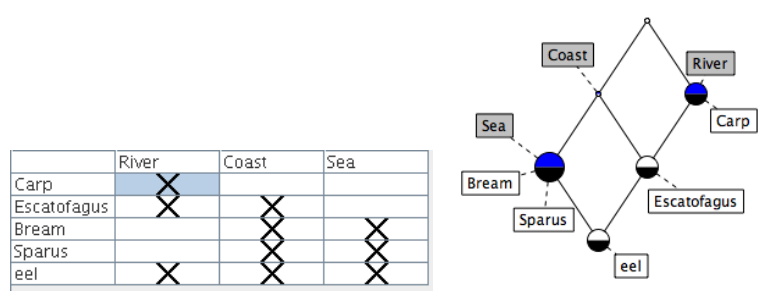

Fig. 1. Formal context on fishes and its concept lattice

devoted to succinctly present FCA. Sect. III and IV describe how to associate concept lattices to CS from observations, and $\mathrm{SFCH}$ is asserted. Other sections are devoted to the analysis of different activities associated to qualitative analysis of CS: forecasting/prediction (Sect. V), catalogation/classification of objects (Sect. VI), Semantic systems (subsystems of Wordnet) in Sect. VI, and to explicitly present the contrast with the topology associated to concept lattices obtained from random formal contexts (Sect. VIII). A final section with conclusions and future work is given.

\section{BACKGROUND: FORMAL CONCEPT ANALYSIS}

FCA mathematizes the philosophical understanding of a concept as a unit of thoughts composed of two parts: the extent and the intent. The extent covers all objects belonging to this concept, while the intent comprises all common attributes valid for all the objects under consideration [10]. It also allows the computation of concept hierarchies from data tables.

A formal context $M=(O, A, I)$ consists of two sets, $O$ (objects) and $A$ (attributes) and a relation $I \subseteq O \times A$. Finite contexts can be represented by a 1-0-table (identifying $I$ with a boolean function on $O \times A$ ). Given $X \subseteq O$ and $Y \subseteq A$ it defines $X^{\prime}=\{a \in A \mid o I a$ for all $o \in X\}$ and $Y^{\prime}=\{o \in$ $O \mid o I a$ for all $a \in Y\}$.

The main goal of FCA is the computation of CL associated with the context. A (formal) concept is a pair $(X, Y)$ such that $X^{\prime}=Y$ and $Y^{\prime}=X$. For example, the concept lattice from formal context about fishes of Fig. 1, left (attributes are understood as "live in") is depicted in Fig. 1, right. Each node is a concept, and its intension (or extension) can be formed by the set of attributes (or objects) included along the path to the top (or bottom). For example, bottom concept ( $\{$ eel $\},\{$ Coast, Sea, River $\})$ is the concept euryhaline fish. CL contains every concept which can be extracted from context, and concept are defined but it is possible there no exist an specific term (word) to denote it.

\section{FCA-BASED BOUNDED REASONING ON CS}

The selection of FCA for processing qualitative information about complex systems lies in the fact that human's reasoning in fact, our Bounded reasoning (BR) skills- about the dynamics and organization of a Complex System has a qualitative nature. Therefore, human reasoning and conjectures about the CS can be expressed in qualitative terms (possibly choosing thresholds and multivalued attributes). Once qualitative hypothesis are presented, even non-symbolic mechanisms for reasoning can

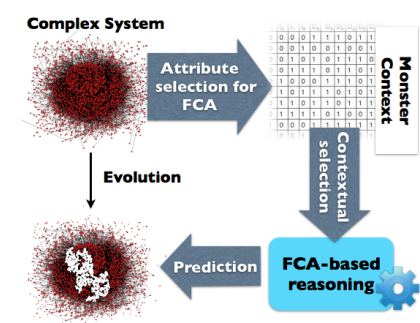

Fig. 2. FCA-based model for qualitative reasoning with Complex Systems

be useful to validate the conjectures. The qualitative reasoning process by means of FCA would be depicted as in Fig. 2. The observer has to select attributes and objects he considers relevant to determine CS dynamics, and the reasoning focuses on the associated subcontext (contextual selection). Then he/she can consider the elements of CS as objects of a formal context. This context (often with a huge size) is built by means of data extraction and processing, expert observations, data mining, etc. It is expected that reasoning with the contextual selection gives some information about the CS. If the the goal is to reason with qualitative features, it is interesting to extract emergent concepts from these interactions. It's here where FCA can play a relevant role. In [5] this approach was applied for reasoning with contextual selections.

The model (described in [2]) is composed of events (objects) which have a number of properties (attributes). They constitute a universal formal context $\mathbb{M}$ (the monster context). Thus $\mathbb{M}$ can be considered as the global memory from which subcontexts are extracted. Once the specific context is considered, it is interesting to consider the knowledge extracted from the formal context (implication basis or association rules [2]).

Particularly interesting is the case of inferring properties about future events when the monster model presents attributes of past events (used in forecasting, Sect. V). When some attributes are known to be satisfied by a future event, the inference process consists of three steps:

- A question on whether a new event (object) has a property (attribute) is raised. Some other properties (attributes) on this object are known $\left\{A_{1}, \ldots A_{n}\right\}$.

- The subcontext induced by a selection of attributes is used to compute a Knowledge Base $\mathcal{L}$ called contextual KB. This $\mathrm{KB}$ consist on a set of implications among attributes, extracted from the subcontext.

- A reasoning system [5] is executed on the contextual KB, taking $\left\{A_{1}, \ldots A_{n}\right\}$ as initial facts. The results are attributes inferred about the object.

Note that it only computes those attributes entailed from the set of attributes selected by the user. Therefore we would need to understand the topology of the lattice to properly choose the attributes to reason with.

\section{SCALE-FREE RESIDUE OF CONCEPT LATTICES}

Given an attribute set for the objects of a CS, the concept lattice associated to the CS (CLCS) is the concept lattice built from the monster context associated to the CS. Note that the 
CLCS can be considered as a directed graph (as Hasse diagram indicates) or as a nondirected graph if necessary. The analysis of CLCS shows interesting concepts for better understanding the structure and dynamics of CS. It is also useful to consider the role some attributes play in the qualitative study of CS [3]. It should be noted that the CLCS is a complex network of semantic relationships which is not bounded by the self language, as in other semantic networks [13], because there are concepts which are not represented by a single language term nor a intelligible definition by the observer. It is expected to face complex networks with extreme structural topology. The complexity of such CLCS lies in the fact that the combinatorial nature of FCA covers every formal concept.

Recall that a scale-free network is one whose degree distribution follows a power law, at least asymptotically: the fraction $\mathrm{P}(\mathrm{k})$ of nodes in the network having $k$ connections to other nodes goes for large values of $\mathrm{k}$ as $P(k) \sim c k^{-\gamma}$ where $c$ is a normalization constant and $\gamma$ is a parameter whose value is typically in the range $2<\gamma<3$, although occasionally it may lie outside these bounds (as we will see below). The asymptotic behavior means that, in practice, few empirical phenomena obey the power law distribution for all the values [8]. It is more common this behavior to appear from a certain threshold $x_{\text {min }}$. The scale-free residue of a CLCS is the set of its nodes whose degree is greater than $x_{\text {min }}$.

We expect that the topological analysis of the dynamics of CLCS shows a big picture of the complex system itself. Normally one will expect that the CLCS has a topology similar to other concept lattices, even similar to concept lattices associated to random formal contexts (with similar density). However, as it will be discussed in the following examples, the degree distribution is not usually very large although there are many attributes in play. As a consequence of this, the lattice is very complex, exhibiting a different topological structure than the lattices extracted from random contexts, for example.

It is possible to refine the choice of $x_{\min }$ [8]. We use $x_{\min }$ as the degree value with the maximum frequency within the CLCS (the maximum of the degree distribution). Lastly, it should be noted that CLCS are not random networks, whose degree distribution follows a Poisson law [1].

The rest of the paper is devoted to test the following

Scale-Free Conceptualization Hypothesis (SFCH):
Only if the attribute set selected to observe the Complex
System is computable, objective and induces a Concept
Lattice which provides a sound analysis of the CS (from the
point of view of BR), then its degree-distribution is scale-free

in a number of CS of different nature.

\section{FORECASTING/PREDICTION}

Two very different CS on which prediction (or diagnosis) are relevant humans' aims have been selected. The first one (Spanish soccer competition) is a CS where a great number of levels, factors and agents play. The goal is to simulate human forecasting on soccer matches [3] (see previous [2], [4], [5]).

\begin{tabular}{|c|c|c|c|c|c|c|c|c|}
\hline & $|\mathbb{O}|$ & $|\mathbb{A}|$ & Density & $|C L|$ & $\langle k>$ & $x_{m}$ & $s c(\%)$ & $\gamma$ \\
\hline $05 / 06$ & 842 & 94 & $13.31 \%$ & 27,434 & 8.27 & 7 & 77.62 & 5.33 \\
\hline $05 / 07$ & 1,684 & 94 & $13.30 \%$ & 81,490 & 9.47 & 9 & 60.87 & 6.08 \\
\hline $05 / 08$ & 2,526 & 94 & $13.26 \%$ & 140,739 & 9.97 & 9 & 68.2 & 6.34 \\
\hline $05 / 09$ & 3,368 & 94 & $13.37 \%$ & 243,959 & 10.62 & 10 & 63.6 & 6.84 \\
\hline $05 / 10$ & 4,210 & 94 & $13.36 \%$ & 324,146 & 10.82 & 10 & 66.20 & 6.69 \\
\hline
\end{tabular}

\begin{tabular}{|l|c|c|c|c|c|}
\hline 3-month intervals & $|\mathbb{O}|$ & $|\mathbb{A}|$ & Density & $|C L|$ & $\langle k\rangle$ \\
\hline 1 & 787 & 81 & $14.73 \%$ & 294 & 5.310 \\
\hline 4 & 3,148 & 81 & $14.63 \%$ & 5,864 & 7.676 \\
\hline 7 & 5,509 & 81 & $13.63 \%$ & 44,911 & 9.595 \\
\hline 10 & 7,870 & 81 & $10.61 \%$ & 62,870 & 9.678 \\
\hline
\end{tabular}

TABLE I

DATA ON ACCUMULATED CLCS FOR SOCCER (UP) AND DARFUR (DOWN).
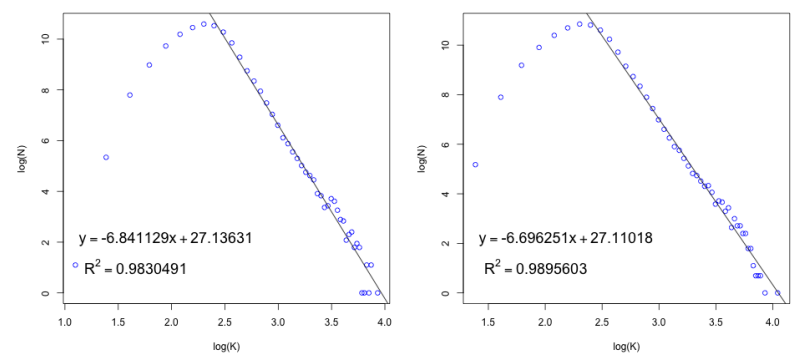

Fig. 3. Log-log degree distribution of CLCS for Sport forecasting (4 and 5 seasons)

The second one is an experimental application on a CS where spatial features are relevant, the Darfur conflict.

\section{A. Complex systems in sport}

The first case represents a nice example of successful application of BR on CS by means of FCA tools. The study of complex systems associated to sport is increasing its importance due to economic (and political) reasons. Several evidences about prediction in betting markets or match forecasting encourage many projects on this application field. In [3] we focus our efforts in soccer results forecasting. In this case study, monster model is built by considering matches as objects and selecting qualitative attributes related to team's features and previous results [4]. The monster context covers -among other information- all matches from season 2005/06 to season 2010/11 of spanish premier league. Attributes express properties of the teams involved in the match, and they are booleanized if it were necessary [4], [2].

Table I shows the main parameters of the cumulative CLCS. Fig. 3 shows the log-log chart representing the scale-free residue behavior.

It is worth to note the growth and size of $\gamma$ in the distributions shown in table I. One explanation for this behavior is based on [6], where the asymptotic behavior of scale-free networks is studied. In the mentioned work, two models of limit behavior are shown. In one of them, the number of nodes in the network remains constant, and the distribution tends to a Gaussian one. In our case, if it supposes the process starts from a node set with $2^{94}$ nodes (the power set of Attribute 

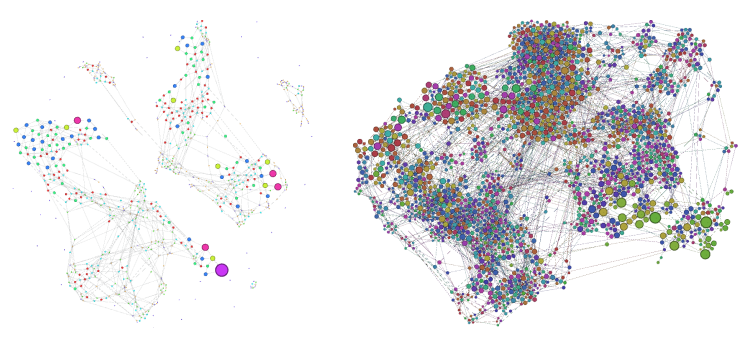

Fig. 4. Deduction graph for Sport Forecasting (left) and for Darfur (right)

set), and in each step new objects are added, new relations in the lattice emerge. From this point of view, the CLCS shows a distribution which is in the middle of the pure scale-free and the Gaussian one [6]. According to SFCH, as closer to the pure scale-free is the CLCS degree distribution, the information on CS dynamics will be richer and more useful.

It is not possible to predict how will be the attribute distribution of new objects (matches), therefore it can only be estimated the complexity of the reasoning process (production system execution) on those (on their initial attributes). In order to understand better the complexity of logical reasoning in a CLCS, it is possible to analyze the topological structure of concept nodes which are involved in frequent deduction tasks. In Fig. 4 (left) the deduction graph of CLCS for Sport Forecasting is depicted. The nodes of the graph are the concepts of CLCS whose intents contains both the left side of at least the $10 \%$ of the rules, and the right side of at least $10 \%$ of the rules in the Knowledge Base extracted from the context. The graph contains 715 nodes and 1296 arcs and its diameter is 7 . The diameter (considered as a directed graph) suggests that a reasonable (upper bound) estimation of rule firings by the production system is 7 (a deduction made by reasoning system induces a path in the CLCS). The existence of many connected components is useful to decide which attributes are relevant to frequently achieve a certain result in prediction (those that appear in the same connected component than the attributes related with the result).

\section{B. Darfur conflict}

The second CS is The Darfur Conflict. The importance and its character of contemporary relatively little-known genocide are the main motivations to work with the information available about this topic. The conflict represents a very complex system, and the information about its dynamics can be collected from different sources (US government, nongovernmental organizations, academic research groups, etc.).

The key feature which differentiates the multilevel nature of this CS from the one above is that the information is harder to process and to compute than in the former example. Excellent references as [12] provide a number of insights on the conflict that may be considered. Also geolocation services allow to consider spatial features of events and interactions in the region. It is worthy to note that the information increases in a different way as in the CS on sport forecasting: multiple resources, heterogeneous data and frequent incorporation of new information resources. In the current state of the project,

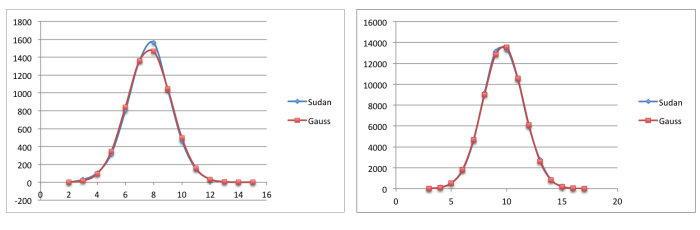

Fig. 5. Degree distributions of Darfur CS (4 and 10 3-month periods) and their Gauss approximations

the analysis uses the already cited [12] and other sources: Aerial military attacks on civilians and humanitarians in Sudan, 1999 - 2011 ${ }^{1}$, Darfur - Destruction of 1,000 Villages ${ }^{2}$, Crisis in Darfur ${ }^{3}$ and North Sudan ${ }^{4}$. Nevertheless, a number of new information sources and data will be considered shortly.

Preliminary experiments with this data showed a significative number of false positives (that is, the system predicts attacks to villages which did not really happen) and a small number of false negatives (something more serious). Two reasons could explain this behavior. On the one hand, the information contained in the data is poor in some cases (even there exist a number of ambiguities), affecting the variety and accuracy of the attributes. The Gauss distribution of nodes degree in the associated CLCS (see Table I and Fig. 5) indicates that there are not key features within the concept population to exploit in the reasoning process. This fact is reinforced when it is found that the cumulative CLCS stabilizes after 10 periods (that is, there are not any new relevant information). This phenomena suggests that to obtain a scale-free distribution more attributes are necessary in order to increase the number of concepts, which is one of the requisites showed in [1] to asymptotically obtain a free-scale network.

On the other hand, from the point of view of the reasoning, the deduction graph for the CLCS shows a very complex network (Fig. 4 (right)), where only one period of the conflict is represented (1986 nodes and 6413 links). Its diameter is 13 and is highly connected, suggesting that it does not provide any information about which attributes are more relevant in order to predict a certain result.

\section{Clasificacion}

To illustrate the SFCH in the descriptive analysis of other systems, four contexts have been selected:

Mushrooms ${ }^{5}$ (context $M$ ): A mushroom dataset, being described in terms of physical characteristics, where objects are mushrooms and attributes are qualitative properties of the mushrooms. The formal context associated to the data can be understood as a qualitative description of the current state of evolution of a kind of vegetables.

\footnotetext{
${ }^{1}$ http://www.sudanbombing.org/

${ }^{2}$ http://bbs.keyhole.com/ubb/ubbthreads.php?ubb=showflat\&Number= 721111\&site_id=1

${ }^{3}$ http://www.ushmm.org/maps/projects/darfur/

${ }^{4}$ http://bbs.keyhole.com/ubb/ubbthreads.php?ubb=showflat\&Number= 393317

${ }^{5}$ http://archive.ics.uci.edu/ml/datasets/Mushroom
} 


\begin{tabular}{|c|c|c|c|c|c|c|c|}
\hline & $|\mathbb{O}|$ & $|\mathbb{A}|$ & Density & $|C L|$ & $\langle k\rangle$ & $x_{\min }$ & $s c(\%)$ \\
\hline$M$ & 8,124 & 119 & $19.33 \%$ & 238,709 & 11.453 & 11 & 63.04 \\
\hline$T^{3}$ & 958 & 29 & $34.48 \%$ & 59,504 & 10.637 & 9 & 67.27 \\
\hline$W$ & 68 & 178 & $20.48 \%$ & 25,408 & 9.370 & 8 & 70.92 \\
\hline$C$ & 1,728 & 25 & $28 \%$ & 12,639 & 9.438 & 7 & 98.96 \\
\hline
\end{tabular}

TABLE II

DATA ON CLCS FOR OTHER CONTEXTS (SEE ALSO FIG. 6)
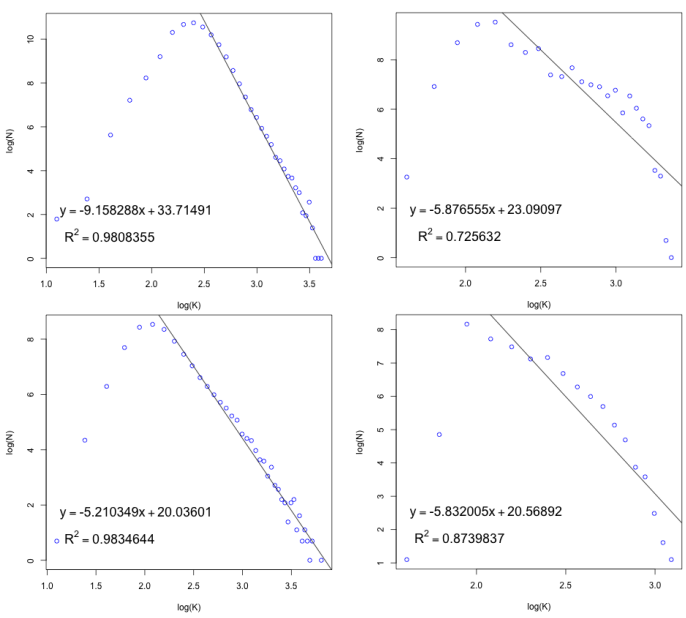

Fig. 6. Log-log degree distribution for Mushroom and $T^{3}$ (up), Wine and Car evaluation contexts (down)

Tic-Tac-Toe $e^{6}$ (or $T^{3}$ ): End results of the tic-tac-toe game. The objects are possible game ends and attributes describe the board configurations at the end of game. It is not a CS.

$W_{i n e}{ }^{7}$ (context $W$ ): A dataset containing different wines (objects) where attributes are their qualitative properties. It could be enriched with more specific attributes related with biological systems associated to its production/processing [7].

Car Evaluation $^{8}$ (context $C$ ): A dataset representing cars acceptability. Objects are cars and attributes are subjective qualitative properties of cars. From the point of view of this paper, the context collects qualitative information about automobile industry, evolution and products.

Wine and Mushroom consist on a human-made cataloguing about the evolution of a CS. Those show a scale-free residue $\left(R^{2}>0.95\right)$. The context $T^{3}$ is not based on classification of a CS, and distribution satisfies $R^{2}<0.75$. Lastly the case of car evaluation context has an intermediate behavior that we conjecture as a consequence of the attempt to describe objects produced within a CS (automobile industrial complex) by means of subjective attributes (opinion), thus contradictory opinions are allowed. This borderline case presents an intermediate degree distribution (Fig. 6) with $0.85<R^{2}<0.9$

\section{WORDNET SUBSYSTEMS}

Another case study considered is WordNet (http://wordnet. princeton.edu/), the lexical database of english where words

\footnotetext{
${ }^{6}$ http://archive.ics.uci.edu/ml/datasets/Tic-Tac-Toe+Endgame

${ }^{7}$ http://archive.ics.uci.edu/ml/datasets/Wine

${ }^{8} \mathrm{http}: / /$ archive.ics.uci.edu/ml/datasets/Car+Evaluation
}
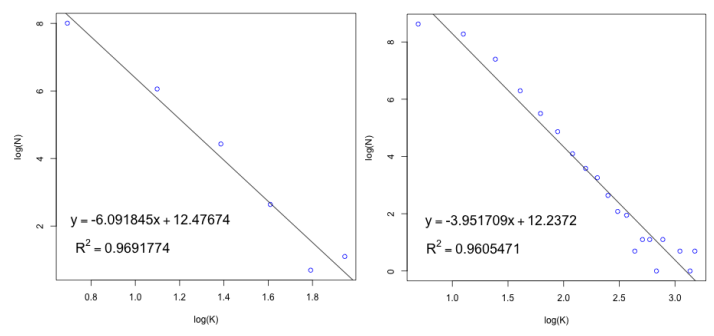

Fig. 7. Log-log degree distribution for Adverbs and verbs subcontexts of WordNet
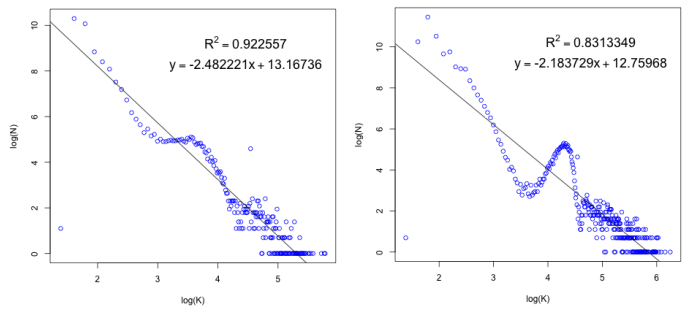

Fig. 8. Two representative examples of Log-log degree distribution of random contexts with density similar to sport forecasting (accumulated for one and two seasons)

are grouped into sets of cognitive synonyms (synsets). Wordnet represents the state-of-art of our own language evolution in such a dimension. To build the formal context, each single word has been considered as an object and each possible synset as an attribute, where an object owns an attribute if the word belongs to the corresponding synset. WordNet, considered as a formal context, produces a concept lattice very similar to the synsets structure. Two smaller word subsets have been considered, adverbs and verbs. In both cases SFCH holds:

\begin{tabular}{|l|c|c|c|c|c|c|}
\hline & $|\mathbb{O}|$ & $|\mathbb{A}|$ & Density & $|C L|$ & $\langle k\rangle$ & $s c(\%)$ \\
\hline Adverbs & 4,481 & 3,621 & $0.03 \%$ & 3,529 & 2.187 & 100 \\
\hline Verbs & 11,529 & 13,767 & $0.02 \%$ & 12,222 & 2.967 & 100 \\
\hline
\end{tabular}

with $x_{\min }=2$ (see Fig. 7). Both cases satisfy SFCH.

\section{RANDOM CONTEXTS}

In order to show how specific is the topology of CLCS, we compare it with that of the CL associated to random contexts. Such contexts represent a set of observations (objects with attributes) on a system whose behavior seems to be random, from the point of view of the selected attribute set. The absence of strong relationship among attributes will be represented in the associated CL. Random contexts (and associated CL) are useful to contrast the SFCH. In order to show whether a scale-free residue exists or not in a certain type of CLCS an experiment was carried out to compare the CLCS with the CL associated to random contexts. In this experiment two sets of 10,000 random formal contexts, with fixed density, number of objects and number of attributes, were generated:

\begin{tabular}{|c|c|c|c|c|}
\hline Dataset & $|\mathbb{O}|$ & $|\mathbb{A}|$ & Density & average $R^{2}$ \\
\hline$m 1$ & 842 & 94 & $13.31 \%$ & 0.847606 \\
\hline$m 2$ & 1,684 & 94 & $13.30 \%$ & 0.8037136 \\
\hline$m 3$ & 2,526 & 94 & $13.26 \%$ & 0.809734 \\
\hline
\end{tabular}

The fixed parameters take the size and dimension values of the two first monster contexts for soccer (see Table I). 


\begin{tabular}{|c|c|c|c|c|c|c|c|c|c|}
\hline System & CS? & Aim & $\begin{array}{l}\text { Objective } \\
\text { Data? }\end{array}$ & $\begin{array}{l}\text { Accuracy } \\
\text { of data? }\end{array}$ & $\begin{array}{l}\text { BR-Modeling } \\
\text { (BR1) }\end{array}$ & $\begin{array}{l}\text { BR-agents } \\
\text { within CS (BR2) }\end{array}$ & $\begin{array}{l}\text { Modeling reasoning of } \\
\text { agents within CS (BR3) }\end{array}$ & $\begin{array}{l}\text { Scale } \\
\text { Free? }\end{array}$ & SFCH? \\
\hline Sport & Yes & Human bet simulation & Yes & Yes & Yes & Yes & Yes & Yes & Yes \\
\hline Darfur & Yes & Prediction & Yes & Poor & Yes & Yes & No & No & Yes \\
\hline Mushroom & Yes & Classification & Yes & Yes & Yes & No & No & Yes & Yes \\
\hline$T^{3}$ & No & Classification & Yes & Yes & No & No & No & No & Yes \\
\hline Wine & Yes & Classification & Yes & Yes & Yes & Yes & No & Yes & Yes \\
\hline Car & No & Evaluation & Medium & No & Yes & Yes & No & No & Yes \\
\hline
\end{tabular}

TABLE III

DESCRIPTION OF THE EXAMPLES USED IN THE PAPER

Results show that the degree distributions of CLCS are very different to the distributions of CL associated to random formal contexts (a random context can be interpreted as the result of the observation of a CS by means of non relevant attributes or a qualitative observation of a chaotic system). In Fig. 8 the degree distribution of CL associated to random formal contexts is shown, and it does not follows a power-law distribution. Also this has been studied by means of a goodness of fit test ( $\chi^{2}$ test), to proof that the degree distribution of CL associated to random formal contexts does not follow the power-law distribution from $x_{m i n}$. Finally it is worthy to note that in the degree distribution of $\mathrm{CL}$ associated to random formal contexts appears a kind of phase transition (see also Fig. 8).

\section{CONCluding Remarks And Future Work}

Throughout the paper a number of CLCS have been studied. Table III shows their main features. It is worthy to note the different uses of BR considered, both within the CS and in its analysis. The first one is its use for modeling the system (extracting a set of recognizable qualitative features for describing phenomena/objects), BR1. The second one represents the use of BR by the self agents which live within the System to study, BR2. The third use of BR, BR3 considers that the system designed uses BR for simulating agents within the CS in order to achieve the goal. The last one is used only in an specific case, where the aim is to simulate an specific behavior within a CS.

The SFCH relates CLCS topology with the information about CS in two interesting CS, (Sport a Darfur conflict). The positive case is deeply analyzed in [3]. The negative one, the case of Darfur Conflict (with Gauss distribution) shows that the use of data with poor quality produces a CLCS with a topology that prevents successful reasoning due to the fact that it does not allow to soundly discriminate target attributes. We are currently adding new attributes and data to the last CS in order to enrich the information.

CLCS strongly depends on the use of specific terms (attributes) as they have direct influence on concept relationships. Since in CL each concept represents the definition of a new term, CLCS should be similar to semantic networks. In [13] the semantic network among concepts expressed by (english language) terms is studied, and it also has a scalefree distribution. In contrast to the aforementioned work, our network is not sparse, because we do not use terms, but concepts (which are specified by an attribute set and not by an isolated term). In [13] $P(k) \sim c k^{-\gamma}$, with $\gamma=3.5$, but in the distribution of CLCS studied here $\gamma>3.5$, suggesting that the scale-free residue has a very small amount of nodes with high connectivity. The network representing words and synonymy relationship between them analyzed in [1] has $\gamma=2.8$. Another example is the semantic network associated to Roget's Thesaurus [14] (see [15]), with $\gamma=3.19$. It remains to study what happens if we expand the language by inserting new language terms defined from the available information.

Future work focuses on finding a relationship between human involvement in CS and degree distribution of CLCS, in order to discover how useful could be the information behind CS to make short-term predictions. Although the experiments show that SFCH is a sound work hypothesis, it is only descriptive. We aim to state strong ties among SFCH and the performance of the reasoning systems designed on the CLCS.

\section{REFERENCES}

[1] R. Albert and A.-L. Barabási, Statistical mechanics of complex networks Reviews of Modern Physics 74, 47-97 (2002).

[2] G. A. Aranda-Corral, J. Borrego-Díaz, J. Galán,Bounded Rationality for Data Reasoning based on Formal Concept Analysis. Proc. DEXA 2011, pp. $350-358$.

[3] G. A. Aranda-Corral, J. Borrego-Díaz, J. Galán, Complex Concept Lattices for Simulating Human Prediction in Sport, to appear in Journal of Systems Science and Complexity (2012)

[4] G. A. Aranda-Corral, J. Borrego-Díaz, J. Galán-Páez, Selecting Attributes for Sport Forecasting using Formal Concept Analysis, ECAL 2011 Workshop on Complex Systems in Sports.

[5] G. A. Aranda-Corral, J. Borrego-Díaz, J. Galán, Confidence-based reasoning with local temporal formal contexts, Proc. 11th Int. Conf. Artif. Neural Networks, LNCS vol. 6692, pp. 461-468 Springer (2011).

[6] A.-L. Barabási, R. Albert, H. Jeong Mean-field theory for scale-free random networks Physica A 272, 173-187 (1999).

[7] A. R. Borneman, P. J. Chambers and I. S. Pretorius Systems Biology as a Platform for Wine Yeast Strain Development in Biology of Microorganisms on Grapes, in Must and in Wine (H. König et al. eds.), 395-414. Springer (2009).

[8] A. Clauset, C.R. Shalizi, and M.E.J. Newman, "Power-law distributions in empirical data" SIAM Review 51(4), 661-703 (2009).

[9] R. Freese, J. Hyndman, and J. B. Nation, Whaley's Theorem for finite lattices, Order 20 (2003), no. 3, 223-228.

[10] B. Ganter and R. Wille. Formal Concept Analysis - Mathematical Foundations. Springer, 1999.

[11] D. G. Goldstein, G. Gigerenzer, Models of ecological rationality: the recognition heuristic, Psychological review, 109(1): 75-90 (2002).

[12] J. Hagan, W. Rymond-Richmond, Darfur and the Crime of Genocide Cambridge University Press, 2008

[13] A. E. Motter, A. P. S. de Moura, Y. Lai, and P. Dasgupta. Topology of the conceptual network of language. Physical Review E, 65, June, 2002.

[14] P.M. Roget, Roget's Thesaurus of EnglishWords and Phrases (1911 ed.). Retrieved June 21, 2012, http://www.gutenberg.org/etext/1068

[15] M. Steyvers, J. B. Tenenbaum, The large-scale structure of semantic networks: statistical analyses and a model of semantic growth, Cognitive Science, 29(1):41-78 (2005) 